\title{
Nasogastric tube feeding: education needs training?
}

\author{
A. L. Jukes, A. M. Abdoolla, S. Galliford, S. Harwood, A. Llewellyn-Edwards and W. N. Magambo \\ Nutrition Support Team, University Hospital of Wales, Cardiff and Vale NHS Trust, CF14 4XW
}

The policy for insertion of a fine-bore nasogastric (NG) feeding tube was developed and implemented by the nutrition support team (NST) in 1999. This enabled the procedure to be undertaken by nurses, a role previously undertaken by doctors. It also introduced $\mathrm{pH}$ measurement of gastric aspirate as an alternative to x-ray to confirm correct position. In order to facilitate the change in practice the NST developed a formal teaching session. The interactive and practical 3-h session is delivered by the multidisciplinary NST and incorporates Trust guidelines for NG tube insertion, confirmation of position, feed and drug administration as well as troubleshooting. A questionnaire to assess baseline knowledge is completed by the staff at the beginning of each session. This consists of ten true/false statements (S1-10) relating to placement, confirmation of position and maintenance of the tube, all of which are discussed during the session. This review aimed to quantify the education undertaken by the NST and to assess its effect on the baseline knowledge of staff within the Trust. Registration and attendance forms in addition to baseline knowledge questionnaires and evaluation reports were reviewed for the sessions held over the 7-year period.

During the 7-year period between June 2000 and May 2007, a total of thirty-five sessions were held, ranging from two to eight sessions per year. Records were missing or incomplete for three of these sessions. 631 staff reserved a place for thirty-three of the thirty five sessions, average nineteen (range eight to twenty-eight) per session. Of those who reserved a place, 63\% (range 8-87\%) attended. An additional 104 attended without prior reservation, average three (range zero to eleven) per session. Overall a total of 504 staff attended sessions during the 7 years, average fifteen (range four to twenty-five) per session. A total of $96 \%$ were nursing staff, $3 \%$ dietitians and $1 \%$ pharmacists. Of staff $84 \%$ were from the acute hospital sites (two), $14 \%$ from outlying hospitals within the Trust (six) and $2 \%$ from primary care.

A total of 443 baseline knowledge questionnaires were completed, average $88 \%$ (range 61-100\%) per session. There has been no overall improvement in baseline staff knowledge despite 7 years of education. Results suggest knowledge of:

the need to confirm tube position (S2), flushing (S9) and occlusion (S10) has been consistently correct more than $80 \%$ of the time, but no overall improvement;

responsibility for insertion (S1) and ability to aspirate fine bore tubes (S4) was variable, correct $25-100 \%$ of the time, no overall improvement;

appropriate removal of guide wire (S3) and use of auscultation (S6) was generally poor but showed overall improvement of 20-40\%; appropriate use of x-ray (S5), syringes (S7) and water (S8) consistently poor and overall reduction of $20 \%$.

The session has been revised and updated during the 7-year period reflecting changes in guidelines, equipment and feedback. Evaluation of the session is excellent and the attendance rate is good.

Disappointingly there has been no overall improvement in the baseline knowledge of the nursing staff, possibly reflecting the size of the Trust, staff turnover and limited resources of the NST. More practical ward-based training is required to consolidate knowledge gained at education session to improve the safety, comfort and overall patient experience of NG feeding. 